\title{
Erratum to: Linguistic neighbourhoods: explaining cultural borders on Wikipedia through multilingual co-editing activity
}

Anna Samoilenko ${ }^{1,2^{*}}$, Fariba Karimi ${ }^{1}$, Daniel Edler ${ }^{3}$, Jérôme Kunegis ${ }^{2}$ and Markus Strohmaier ${ }^{1,2}$

\section{"Correspondence:}

anna.samoilenko@gesis.org

${ }^{1}$ GESIS - Leibniz-Institute for the

Social Sciences, 6-8 Unter

Sachsenhausen, Cologne, 50667 Germany

${ }^{2}$ University of Koblenz-Landau, Koblenz, Germany

Full list of author information is available at the end of the article
Unfortunately, the original version of this article [1] contained an error. Within the caption of Table 2 the sentence 'The combination of all hypotheses explains most of the variation in the data (20\%)' should have read 'The combination of all hypotheses explains most of the variation in the data (15\%).' Table 2 has been corrected in the original article and is also included correctly below.

Table 2 MRQAP decomposition of pairwise correspondence between concept co-occurrence and cultural factors

\begin{tabular}{|c|c|c|c|c|c|c|c|c|c|c|}
\hline \multicolumn{2}{|c|}{ Model } & \multirow{2}{*}{$\begin{array}{c}\text { Bilinguals } \\
0.0688\end{array}$} & \multirow{2}{*}{$\begin{array}{c}\text { Lang. family } \\
0.1074\end{array}$} & \multirow{2}{*}{$\begin{array}{c}\text { Religion } \\
0.0900\end{array}$} & \multirow{2}{*}{$\begin{array}{r}\text { Gravity } \\
0.0470\end{array}$} & \multirow{2}{*}{$\begin{array}{l}\text { Distance }^{1} \\
-0.0042^{*}\end{array}$} & \multirow{2}{*}{$\begin{array}{l}R^{2} \text { adj. } \\
0.1458\end{array}$} & \multirow{2}{*}{$\begin{array}{l}\boldsymbol{F} \text {-stat. } \\
410.3\end{array}$} & \multirow{2}{*}{$\begin{array}{l}\text { df } \\
11,984\end{array}$} & \multirow{2}{*}{$\frac{\text { Intercept }}{0.0066}$} \\
\hline 1 & $\begin{array}{l}\text { Estimate } \\
\text { t-statistic }\end{array}$ & & & & & & & & & \\
\hline 2 & $\begin{array}{l}\text { Estimate } \\
\text { t-statistic }\end{array}$ & $\begin{array}{r}0.0676 \\
29.1517\end{array}$ & $\begin{array}{r}0.1075 \\
23.6428\end{array}$ & $\begin{array}{r}0.0894 \\
13.4200\end{array}$ & $\begin{array}{r}0.0464 \\
10.1893\end{array}$ & $\begin{array}{l}- \\
-\end{array}$ & 0.1458 & 512.4 & 11,985 & 0.0067 \\
\hline 3 & $\begin{array}{l}\text { Estimate } \\
\text { t-statistic }\end{array}$ & $\begin{array}{r}0.0703 \\
28.1932\end{array}$ & $\begin{array}{r}0.1129 \\
24.8853\end{array}$ & $\begin{array}{r}0.1022 \\
15.4831\end{array}$ & $\begin{array}{l}- \\
-\end{array}$ & $\begin{array}{l}-0.0009^{*} \\
-0.2989^{*}\end{array}$ & 0.1384 & 482.3 & 11,985 & 0.0067 \\
\hline 4 & $\begin{array}{l}\text { Estimate } \\
t \text {-statistic }\end{array}$ & $\begin{array}{r}0.0685 \\
27.3119\end{array}$ & $\begin{array}{r}0.1080 \\
23.5817\end{array}$ & $\begin{array}{l}- \\
-\end{array}$ & $\begin{array}{r}0.0581 \\
12.7773\end{array}$ & $\begin{array}{l}-0.0016^{*} \\
-0.5225^{*}\end{array}$ & 0.1329 & 460.5 & 11,985 & 0.0074 \\
\hline 5 & $\begin{array}{l}\text { Estimate } \\
\text { t-statistic }\end{array}$ & $\begin{array}{r}0.0716 \\
28.1697\end{array}$ & $\begin{array}{l}- \\
-\end{array}$ & $\begin{array}{r}0.0916 \\
13.4180\end{array}$ & $\begin{array}{r}0.0598 \\
12.8396\end{array}$ & $\begin{array}{l}-0.0055^{*} \\
-1.7256^{*}\end{array}$ & 0.1061 & 356.9 & 11,985 & 0.0075 \\
\hline 6 & $\begin{array}{l}\text { Estimate } \\
\text { t-statistic }\end{array}$ & $\begin{array}{l}- \\
-\end{array}$ & $\begin{array}{r}0.1134 \\
24.2095\end{array}$ & $\begin{array}{r}0.0881 \\
12.7958\end{array}$ & $\begin{array}{r}0.0546 \\
11.5815\end{array}$ & $\begin{array}{l}0.0272 \\
9.0453\end{array}$ & 0.09140 & 302.5 & 11,985 & 0.0070 \\
\hline 7 & $\begin{array}{l}\text { Estimate } \\
t \text {-statistic }\end{array}$ & $\begin{array}{r}0.0700 \\
30.2487\end{array}$ & $\begin{array}{r}0.1129 \\
24.8885\end{array}$ & $\begin{array}{r}0.1020 \\
15.5098\end{array}$ & $\begin{array}{l}- \\
-\end{array}$ & $\begin{array}{l}- \\
-\end{array}$ & 0.1386 & 643.1 & 11,986 & 0.0067 \\
\hline 8 & $\begin{array}{l}\text { Estimate } \\
t \text {-statistic }\end{array}$ & $\begin{array}{r}0.0703 \\
27.9237\end{array}$ & $\begin{array}{r}0.1151 \\
25.1460\end{array}$ & $\begin{array}{l}- \\
-\end{array}$ & $\begin{array}{l}- \\
-\end{array}$ & $\begin{array}{l}0.0030^{*} \\
0.9388^{*}\end{array}$ & 0.1212 & 552.2 & 11,986 & 0.0076 \\
\hline 9 & $\begin{array}{l}\text { Estimate } \\
\text { t-statistic }\end{array}$ & $\begin{array}{l}- \\
-\end{array}$ & $\begin{array}{l}- \\
-\end{array}$ & $\begin{array}{r}0.0898 \\
12.7323\end{array}$ & $\begin{array}{r}0.0684 \\
14.2619\end{array}$ & $\begin{array}{l}0.0272 \\
8.8191\end{array}$ & 0.0470 & 198.2 & 11,986 & 0.0079 \\
\hline 10 & $\begin{array}{l}\text { Estimate } \\
\text { t-statistic }\end{array}$ & $\begin{array}{r}0.0700 \\
29.5521\end{array}$ & $\begin{array}{l}- \\
-\end{array}$ & $\begin{array}{r}0.0909 \\
13.3370\end{array}$ & $\begin{array}{r}0.0590 \\
12.7297\end{array}$ & $\begin{array}{l}- \\
-\end{array}$ & 0.1060 & 474.8 & 1,986 & 0.0075 \\
\hline 11 & $\begin{array}{l}\text { Estimate } \\
\text { t-statistic }\end{array}$ & $\begin{array}{l}- \\
-\end{array}$ & $\begin{array}{r}0.1140 \\
24.1755\end{array}$ & $\begin{array}{l}- \\
-\end{array}$ & $\begin{array}{r}0.0654 \\
13.9808\end{array}$ & $\begin{array}{l}0.0296 \\
9.7791\end{array}$ & 0.0790 & 344.0 & 11,986 & 0.0077 \\
\hline 12 & $\begin{array}{l}\text { Estimate } \\
\text { t-statistic }\end{array}$ & $\begin{array}{r}0.0712 \\
30.4703\end{array}$ & $\begin{array}{r}0.1151 \\
25.1430\end{array}$ & $\begin{array}{l}- \\
-\end{array}$ & $\begin{array}{l}- \\
-\end{array}$ & $\begin{array}{l}- \\
-\end{array}$ & 0.1212 & 827.8 & 11,987 & 0.0076 \\
\hline 13 & $\begin{array}{l}\text { Estimate } \\
t \text {-statistic }\end{array}$ & $\begin{array}{r}0.0738 \\
28.6184\end{array}$ & $\begin{array}{l}- \\
-\end{array}$ & $\begin{array}{l}- \\
-\end{array}$ & $\begin{array}{l}- \\
-\end{array}$ & $\begin{array}{l}0.0027 \\
0.8295\end{array}$ & 0.0749 & 486.5 & 11,987 & 0.0085 \\
\hline
\end{tabular}

(c) 2016 Samoilenko et al. This article is distributed under the terms of the Creative Commons Attribution 4.0 International License (http://creativecommons.org/licenses/by/4.0/), which permits unrestricted use, distribution, and reproduction in any medium, provided you give appropriate credit to the original author(s) and the source, provide a link to the Creative Commons license, and indicate if changes were made. 
Table 2 (Continued)

\begin{tabular}{|c|c|c|c|c|c|c|c|c|c|c|}
\hline Mo & del & Bilinguals & Lang. family & Religion & Gravity & Distance $^{1}$ & $R^{2}$ adj. & $F$-stat. & df & Intercept \\
\hline 14 & $\begin{array}{l}\text { Estimate } \\
\text { t-statistic }\end{array}$ & $\begin{array}{l}- \\
-\end{array}$ & - & - & $\begin{array}{r}0.0794 \\
16.7162\end{array}$ & $\begin{array}{l}0.0296 \\
9.5508\end{array}$ & 0.0342 & 213.4 & 11,987 & 0.0086 \\
\hline 15 & $\begin{array}{l}\text { Estimate } \\
\text { t-statistic }\end{array}$ & $\begin{array}{r}0.0733 \\
30.9368\end{array}$ & - & $\begin{array}{r}0.1072 \\
15.9020\end{array}$ & $\begin{array}{l}- \\
-\end{array}$ & - & 0.0940 & 622.8 & 11,987 & 0.0076 \\
\hline 16 & $\begin{array}{l}\text { Estimate } \\
\text { t-statistic }\end{array}$ & $\begin{array}{l}- \\
-\end{array}$ & $\begin{array}{r}0.1222 \\
25.9063\end{array}$ & - & $\begin{array}{l}- \\
-\end{array}$ & $\begin{array}{r}0.0357 \\
11.8512\end{array}$ & 0.0641 & 411.6 & 11,987 & 0.0080 \\
\hline 17 & $\begin{array}{l}\text { Estimate } \\
\text { t-statistic }\end{array}$ & $\begin{array}{l}- \\
-\end{array}$ & - & $\begin{array}{r}0.0936 \\
13.2534\end{array}$ & $\begin{array}{r}0.0741 \\
15.5280\end{array}$ & - & 0.0409 & 256.8 & 11,987 & 0.0080 \\
\hline 18 & $\begin{array}{l}\text { Estimate } \\
t \text {-statistic }\end{array}$ & $\begin{array}{l}- \\
-\end{array}$ & - & - & $\begin{array}{l}- \\
-\end{array}$ & $\begin{array}{r}0.0372 \\
12.0025\end{array}$ & 0.0118 & 144.1 & 11,988 & 0.0090 \\
\hline 19 & $\begin{array}{l}\text { Estimate } \\
\text { t-statistic }\end{array}$ & $\begin{array}{l}- \\
-\end{array}$ & - & - & $\begin{array}{r}0.0861 \\
18.2514\end{array}$ & - & 0.0269 & 333.1 & 11,988 & 0.0087 \\
\hline 20 & $\begin{array}{l}\text { Estimate } \\
\text { t-statistic }\end{array}$ & $\begin{array}{l}- \\
-\end{array}$ & - & $\begin{array}{r}0.1144 \\
16.3447\end{array}$ & $\begin{array}{l}- \\
-\end{array}$ & - & 0.0217 & 267.1 & 11,988 & 0.0081 \\
\hline 21 & $\begin{array}{l}\text { Estimate } \\
t \text {-statistic }\end{array}$ & $\begin{array}{l}- \\
-\end{array}$ & $\begin{array}{r}0.1233 \\
25.9798\end{array}$ & - & $\begin{array}{l}- \\
-\end{array}$ & - & 0.0532 & 674.9 & 11,988 & 0.0081 \\
\hline 22 & $\begin{array}{l}\text { Estimate } \\
\text { t-statistic }\end{array}$ & $\begin{array}{r}0.0746 \\
31.1808\end{array}$ & - & - & $\begin{array}{l}- \\
-\end{array}$ & - & 0.0749 & 972.2 & 11,988 & 0.0085 \\
\hline
\end{tabular}

${ }^{1}$ Primary language.

The combination of all hypotheses explains most of the variation in the data (15\%). The most plausible explanations are the number of bilinguals and shared religion. The results of MRQAP agree with the ranking of hypotheses by the HypTrails algorithm. All statistics except those labelled with * are significant at the 0.05 level.

\section{Author details}

${ }^{1}$ GESIS - Leibniz-Institute for the Social Sciences, 6-8 Unter Sachsenhausen, Cologne, 50667, Germany. ${ }^{2}$ University of Koblenz-Landau, Koblenz, Germany. ${ }^{3}$ Integrated Science Lab, Department of Physics, Umeå University, Umeå, Sweden.

Received: 23 March 2016 Accepted: 23 March 2016 Published online: 08 April 2016

\section{References}

1. Samoilenko A, Karimi F, Edler D, Kunegis J, Strohmaier M (2016) Linguistic neighbourhoods: explaining cultural borders on Wikipedia through multilingual co-editing activity. EPJ Data Sci 5:9. doi:10.1140/epjds/s13688-016-0070-8

\section{Submit your manuscript to a SpringerOpen ${ }^{\circ}$ journal and benefit from:}

- Convenient online submission

- Rigorous peer review

- Immediate publication on acceptance

- Open access: articles freely available online

- High visibility within the field

- Retaining the copyright to your article

Submit your next manuscript at $>$ springeropen.com 